\title{
Muerte fetal por infección bacteriana ascendente. Método diagnóstico, una revisión narrativa. ¿Por qué el método que incluye estudio placentario, evaluación de datos clínicos y de laboratorio es eficiente en identificar la infección bacteriana ascendente como causa de muerte fetal?
}

Fetal death from ascending bacterial infection. Diagnostic method, a narrative review. Why is the method that includes placental study, evaluation of clinical and laboratory data efficient in identifying ascending bacterial infection as the cause of fetal death?

Alfredo Ovalle S. ${ }^{1}$ y Elena Kakarieka W. ${ }^{2}$

'Servicio y Departamento de Obstetricia, Ginecología y Neonatología, Hospital Clínico San Borja Arriarán, Santiago, Chile. Facultad de Medicina, Universidad de Chile. Santiago, Chile.

${ }^{2}$ Servicio de Anatomía Patológica, Hospital San Borja Arriarán. Santiago, Chile.

Los autores no tienen conflicto de interés.

El manuscrito no requirió financiamiento.

Recibido: (segunda versión) 25 de mayo de 2021 / Aceptado: 25 de mayo de 2021

\section{Resumen}

Esta revisión narrativa incluye estudios publicados sobre métodos de clasificación de mortinatos y su eficiencia para identificar la infección bacteriana ascendente (IBA) como causa de muerte fetal (MF), mediante búsqueda en PubMed, Cochrane, Embase, ScienceDirect, Wiley Online Library, Scielo. Muchos niños mueren antes de nacer en todo el mundo y no se ha logrado reducir la MF porque los métodos empleados no han sido los adecuados y porque no se diagnostica la IBA, la causa más frecuente de MF en un hospital público de Chile. Los sistemas que utilizan los datos clínicos, de laboratorio y estudio placentarios, INCODE, CORM, son los más eficientes para identificar la IBA como origen de la MF. Se ha demostrado que los marcadores específicos de infección/inflamación placentaria, corioamnionitis histológica/funisitis aguda son de mayor eficiencia para diagnosticar la IBA que la autopsia fetal, que el cultivo de líquido amniótico es más eficiente que el cultivo de la placenta para detectar invasión microbiana de la cavidad amniótica y que la muestra de sangre de cordón es eficiente para el diagnóstico etiológico de la infección. El conocimiento de la IBA como causa inicial de MF, ayuda a elaborar guías y normas de prevención de la MF por esta condición.

Palabras clave: muerte fetal; infección bacteriana ascendente; métodos de clasificación de mortinatos.

\section{Abstract}

This narrative review includes published studies of stillbirth classification methods and their efficiency in identifying ascending bacterial infection (ABI), as a cause of fetal death (FD), by searching PubMed, Cochrane, Embase, ScienceDirect, Wiley Online Library, Scielo. Many children die before birth around the world and it has not been possible to reduce FD because the methods used have not been adequate and because $\mathrm{ABI}$, the most frequent cause of FD in a public hospital in Chile, is not diagnosed. Systems using clinical, laboratory and placental study data, INCODE, CORM, are more efficient in identifying ABI as the origin of FD. Specific markers of infection/placental inflammation, histologic chorioamnionitis/acute funitis have been shown to be more efficient in diagnosing ABI than fetal autopsy, that amniotic fluid culture is more efficient than placental culture for detect microbial invasion of the amniotic cavity and that the cord blood sample is efficient for the etiological diagnosis of the infection. The knowledge of the ABI as the initial cause of FD helps to develop guidelines and norms for preventing FD due to this condition.

Keywords: stillbirth; ascending bacterial infection; fetal death classification methods. 
ascendentes desde la vagina, infecciones transplacentarias (ITP), como virales (infección por citomegalovirus, parvovirus B19 y herpes simplex), parasitarias (toxoplasmosis, malaria) y bacterianas (sífilis, infección por Listeria monocytogenes, bacterias de la enfermedad periodontal $)^{3-5}$; diagnósticos microbiológicos variados y cultivos bacterianos tomados de sitios diferentes (placenta, feto y líquido amniótico) que no han demostrado necesariamente causalidad $^{6}$; y microorganismos diversos responsables de la muerte donde el estudio fetal no demuestra predomino de alguno de ellos ${ }^{4}$.

Los sistemas de clasificación de MF tradicionales, son de poca utilidad porque en su mayoría reportan alta proporción de muertes sin explicación ${ }^{7-11}$. Actualmente existen varios métodos, destinados a disminuir las muertes inexplicables, entre ellas la infección como causa de MF, como, Goldenberg ${ }^{12}$, INCODE ${ }^{13}$ y CORM $^{2,5}$ del HCSBA, que utilizan datos clínicos, pruebas diagnósticas específicas para infección, autopsia fetal y estudio de patología placentaria; todos ellos tienen mayor eficacia que los métodos tradicionales para diagnosticar la infección como causa del mortinato.

En el HCSBA la condición más frecuente de MF es la infección bacteriana ascendente (IBA), presente en uno de cada cuatro óbitos ${ }^{2,5}$. El método CORM (Condición Obstétrica Relevante de la Muerte fetal) que emplea la biopsia placentaria, los datos clínicos y de laboratorio, ha demostrado eficacia en la identificación de la MF por $\mathrm{IBA}^{2,5}$.

Se pretende demostrar con esta revisión narrativa, que el método que utiliza el análisis de los datos clínicos, de laboratorio y el estudio placentario, es más eficiente que otros métodos para diagnosticar la MF por IBA- condición frecuente de MF- lo que permitiría diseñar estrategias adecuadas de prevención.

\section{Frecuencia de la MF por infección}

- Con métodos convencionales de clasificación. Varía entre 1 y $7 \%$ en los diferentes estudios de series con muerte perinatal: Galan-Roosen ${ }^{9}$, IBA: 2,5\% (6/239), ITP: 4,3\% (10/239); Recode ${ }^{10}$, infección: $6,5 \%$ (170/2625); PSANZ-PDC infección: 7,2\% (22/306) y Tulip ${ }^{11}$ serie con muertes fetales, IBA: $1 \%(4 / 411)$, ITP: $0,5 \%(1 / 411)$.

- Con métodos que ocupan la causa inicial o primaria de la muerte. La frecuencia de detección aumenta. Varía entre 11 y $26 \%$ en los diferentes estudios: INCODE ${ }^{4}$ : 11,1\% (57/512); Soweto Sud Africa ${ }^{14}: 16 \%(47 / 298)$ y Ovalle: $16,1 \%(45 / 279)^{15}, 26,3 \%(68 / 258)^{5}, 24,9 \%$ $(119 / 479)^{2}$. La frecuencia de la ITP es entre 2 y $3,5 \%$, INCODE: $1,8 \%(9 / 512)^{4}$; Ovalle: $2,1 \%(6 / 279)^{15} ; 3,5 \%$ $(9 / 258)^{5} ; 3,1 \%(15 / 479)^{2}$. La IBA representa $89 \%$ de los casos de MF por infección en el HCSBA ${ }^{2}$. 


\section{¿Por qué los métodos convencionales de clasificación de MF son ineficientes para identificar la muerte por IBA?}

Estos sistemas, que utilizan preferentemente la autopsia, y en casos puntuales la placenta para determinar la causa final de la muerte, son de poca utilidad porque en su mayoría reportan alta proporción de muertes inexplicables y baja proporción por IBA $^{7-11}$.

\section{Método de Galan-Roosen ${ }^{8,9}$}

En uso en Holanda, basado en la causa subyacente de la muerte, ofrece seis causas fundamentales de MF: trauma del parto, infección, patología de la placenta o del cordón, isoinmunización, malformación congénita fetal y complicaciones del parto prematuro pre viable. En una serie prospectiva de 239 casos de muerte perinatal, la infección se encontró en 6,8\%: IBA 2,5\%, ITP 4,3\%.

Comentario. Este sistema es poco eficiente para diagnosticar MF por IBA porque se comete el error de considerar que esta muerte ocurre exclusivamente por infección fetal y no por otros mecanismos. Además, es complejo asignar como causa fundamental a algunas de las categorías de MF escogidas por los autores, como trauma en el parto, patología de la placenta y complicaciones del parto prematuro $(\mathrm{PP})$ pre viable, porque estas condiciones pueden asociarse con variadas patologías originarias de $\mathrm{MF}$, incluyendo IBA.

\section{Método Tulip ${ }^{11}$}

Usa un sistema elaborado por un panel de expertos y contempla una clasificación compuesta por grupos de causas y mecanismos de muerte a través del análisis de los eventos relacionados con la muerte. La clasificación consta de seis causas principales con sub clasificaciones anomalía congénita (cromosómica, síndrome y de órgano o sistema único o múltiple; placenta (lecho placentario, patología placentaria, complicación del cordón umbilical y no especificada; prematuridad (rotura prematura de membranas-RPM antes del parto, PP, disfunción cervical, iatrogénica y no especificada); infección (transplacentaria, ascendente, neonatal y no especificada); otros (hidrops fetal de origen desconocido, enfermedad materna, trauma y otras) y desconocida. Además, se elaboraron seis mecanismos: insuficiencia cardiovascular/circulatoria, insuficiencia multiorgánica, insuficiencia respiratoria, insuficiencia cerebral, insuficiencia placentaria y desconocida. La IBA como causa de MF ocurrió en 1\%, y la ITP en $0,5 \%$.

Comentario. Aunque los autores califican esta clasificación como inequívoca de causa subyacente y de mecanismo de mortalidad perinatal, con fácil acuerdo entre evaluadores, con bajo porcentaje de causas desconocidos, y fácilmente aplicable por un equipo de médicos cuando se siguen las pautas descritas, nuestra opinión es diferente. Este, es un método de clasificación de mortinatos muy elaborado con causas principales y mecanismos fisiopatológicos, lo que lo transforma en un sistema engorroso y difícil de reproducir. Al igual que el método de Galan Roosen, las condiciones prematuridad y placentarias, así como también el mecanismo fisiopatológico de muerte por hipoxia, tal vez el más frecuente, tienen diferentes orígenes, uno de ellos es la IBA que se diagnostica con adecuado estudio placentario y con cultivos microbianos específicos.

Estos dos métodos analizados son de baja utilidad para prevenir MFs futuras.

Revisión sistemática Cochrane ${ }^{16}$. Ratifica esta conclusión. En el manuscrito sobre intervenciones para investigar e identificar las causas de la MF concluye: "Actualmente, en los ensayos controlados aleatorios no hay evidencia en la efectividad de las intervenciones existentes para investigar e identificar las causas de la MF. Debido a la ausencia de ensayos adecuados, esta revisión no puede sugerir recomendaciones clínicas para investigar las causas especificas de los mortinatos"

\section{Factores infecciosos responsables de los resultados adversos perinatales y participación de estos factores en el método que identifica la IBA como causa primaria de muerte}

La causa primaria o inicial del óbito es la entidad fisiopatológica demostrable que inicia la cadena de eventos que conduce a la MF. Estos métodos, utilizan fundamentalmente la biopsia placentaria, con los datos clínicos y de laboratorio. Detectan mayor frecuencia de infección como causa de MF y reducen el número de causas inexplicables. La mayor frecuencia de infección cérvicovaginal/invasión microbiana de la cavidad amniótica (ICV/IMCA) se presenta en series de PP con RPM.

\section{Evidencias}

- Infección cérvicovaginal/invasión microbiana de la cavidad amniótica

En serie ${ }^{17}$ de 80 pacientes con RPM de pre término (PT) entre 24 y 34 semanas de embarazo, sin infección clínica y sin trabajo de parto, manejadas con conducta expectante y con cultivo microbiológico de líquido amniótico (LA) obtenido por amniocentesis transabdominal y de muestras cérvicovaginales, se encontró que todas las IMCA tuvieron ICV y las bacterias aisladas de la IMCA son similares a las encontradas en la ICV. La IMCA se diagnosticó con cultivo positivo de LA. La ICV se diagnosticó con presencia de vaginosis bacteriana (VB) o de vaginitis aeróbica (VA) o de micoplasmas genitales, asociadas con un aumento en el recuento de leucocitos 
( $p<0,01)$. Los niños con funisitis vs niños sin funisitis tuvieron mayor frecuencia de muerte neonatal 29,2 vs $6,7 \%$ e infección del neonato 29,2 vs $6,7 \%(p<0,05)$. La funisitis es marcador de gravedad de infección y de mortalidad neonatal por infección.

En una serie China ${ }^{21}$ con 14.166 partos únicos, se incluyeron 2.372 pacientes en que se sospechó corioamnionitis en el tercer trimestre del embarazo y que tuvieron estudio histológico placentario. Se encontró infección/ inflamación en 15,7\% (373/2.372) de las placentas enviadas a estudio histológico $(15 \% \mathrm{CH}, 0,7 \%$ corioamnionitis clínica). Estos casos se dividieron en cuatro grupos: $\mathrm{CH}$, corioamnionitis clínica $+\mathrm{CH}$, corioamnionitis clínica y control $\sin \mathrm{CH}$. La incidencia de $\mathrm{CH}$ aguda fue de 2,5\% $(355 / 14.166)$ en estos nacimientos. El grupo con $\mathrm{CH}$ en comparación con el grupo control, tuvo edad gestacional $<$ de 34 semanas al parto, sufrimiento fetal y sepsis de inicio temprano en los $\mathrm{RN}(\mathrm{p} \leq 0,01)$. En los subgrupos muy prematuros del grupo de $\mathrm{CH}$, se encontraron más MFs e ingresos más frecuentes a la UCI neonatal que en el grupo control $(p<0,001)$. Recomiendan que el examen histológico placentario deba realizarse de forma rutinaria en partos muy prematuros porque permiten evaluar mejor el resultado perinatal. Se concluye que $\mathrm{CH} /$ funisitis aguda se relacionan con morbilidad neonatal y mortalidad perinatal en pacientes, con RPM de PT y con PP y sospecha de corioamnionitis.

\section{- Virulencia bacteriana}

En una serie de PP con RPM ${ }^{20}$ entre 24 y 34 semanas de embarazo, con 96 pacientes incluidas, referida anteriormente, los neonatos de madres con IMCA por $S$. agalactiae, o por $F$. nucleatum o por $H$. influenzae tuvieron más frecuentemente, infección (36\%), asfixia (36\%), ingreso a UCI neonatal (100\%) y muerte (46\%) que los neonatos de pacientes con IMCA por otras bacterias, únicas o asociadas o IMCA por Ureaplasma urealyticum como único agente etiológico y que hijos de mujeres sin infección $(p<0,01)$. Estos resultados adversos neonatales encontrados en la infección intraamniótica por $S$. agalactiae o por $F$. nucleatum o por $H$. influenzae son expresiones de bacterias de mayor agresividad.

Streptococcus agalactiae (usualmente referido como Streptococcus grupo B) es una bacteria grampositiva de alta frecuencia. Forma parte de la microbiota intestinal distal del ser humano y coloniza frecuentemente el aparato genital inferior de la mujer. Se asocia con infección grave perinatal (sepsis neonatal, neumonía congenita-connatal, muerte perinatal) y secuelas neurológicas ${ }^{22-24}$. En el HCSBA es la bacteria más frecuente asociada con morbilidad/mortalidad perinatal.

Fusobacterium nucleatum es una bacteria anaerobia gramnegativa que habita la cavidad oral. Se asocia con enfermedad periodontal ${ }^{25}$. A pesar de que la evidencia 
mayoritariamente señala que la ruta que sigue la enfermedad periodontal para infectar la cavidad amniótica es hematógena transplacentaria, existe evidencia que también puede ser por IBA, a partir de microbiota oral de la pareja ${ }^{26}$. Se asocia con morbilidad/mortalidad perinatal.

Haemophilus influenzae es un bacilo gramnegativo pequeño y uno de los agentes etiológicos más importantes de meningitis ${ }^{27}$. Se postula que la infección intrauterina fue secundaria a infección ascendente desde la vagina, pues esta bacteria se aisló en el tracto genital inferior de estas mujeres embarazadas ${ }^{28}$. Se asocia con morbilidad/ mortalidad perinatal.

Otras bacterias causantes de morbilidad/mortalidad neonatal aisladas de la cavidad amniótica de pacientes con RPM en el HCSBA son Escherichia coli ${ }^{29}$ y Morganella morganii ${ }^{30}$ Las placentas demostraron presencia de $\mathrm{CH}$ $\mathrm{y}$ de funisitis.

Se concluye que, en la IMCA de pacientes con RPM de PT, existen bacterias de mayor agresividad como $S$. agalactiae, $F$. nucleatum, $H$. influenzae, que se asocian con mayor frecuencia y gravedad de resultados adversos neonatales.

\section{- Síndrome de respuesta fetal inflamatoria (SRFI)}

Se sabe que la IMCA, produce en el feto aumento de citoquinas, entre otros mediadores inflamatorios, que conduce a una injuria orgánica multisistémica asociada con rápida morbimortalidad neonatal, y en algunos casos, con daños neurológicos posteriores (leucomalacia periventricular, hemorragia intraventricular, parálisis cerebral), con enfermedad crónica pulmonar y con enterocolitis necrosante ${ }^{31-33}$.

Los dramáticos resultados adversos obstétricos neonatales encontrados en la IMCA por $S$. agalactiae, $F$. nucleatum o $H$. influenzae en el estudio referido anteriormente, pese al uso de antimicrobianos, permiten plantear la hipótesis que el mayor daño en estos casos, además de la mayor virulencia, podría producirse por incremento de la respuesta fetal inflamatoria ante estos microorganismos.

Esta afirmación se puede sostener por los siguientes hallazgos encontrados en este estudio, que se correlacionan con resultados sobre SRFI, publicados: la corioamnionitis clínica se presentó en la mitad de los casos de IMCA por $S$. agalactiae, $F$. nucleatum o $H$. influenzae y se ha demostrado que la concentración de Il-6 se eleva en la vena umbilical del RN cuya madre tiene corioamnionitis clínica $^{34}$; la $\mathrm{CH}$ apareció en todas las placentas y la funisitis fue más frecuente en las mujeres embarazadas con estas bacterias de cepas agresivas y se ha demostrado que la funisitis es manifestación histológica del $\mathrm{SRFI}^{35}$; en las mujeres con IMCA por $S$. agalactiae, $F$. nucleatum o $H$. influenzae, el intervalo admisión-parto fue más breve y los partos dentro de las $48 \mathrm{~h}$ y dentro de los siete días desde la admisión, fueron significativamente más frecuentes, que en las pacientes con infección por otras bacterias únicas o asociadas, o por $U$. urealyticum y, que en las mujeres embarazadas sin infección, y se sabe que el aumento de la respuesta fetal sistémica de citoquinas proinflamatorias en mujeres con IMCA es seguido por el desencadenamiento inmediato del parto prematuro ${ }^{36}$.

\section{¿En qué consiste el Método CORM (Condición Obstétrica Relevante de Muerte fetal) del HCSBA ? $^{2,5}$}

Este sistema emplea la biopsia placentaria, los datos clínicos y de laboratorio para identificar a la IBA como causa de MF. Es clasificable como IBA el embarazo con alguno de los siguientes requisitos:

- Datos clínicos: condiciones obstétricas en que frecuentemente existe IMCA: corioamnionitis clíni$\mathrm{ca}^{18}$, RPM PT, (IMCA 54\%) ${ }^{17-20}$, sangrado vaginal "idiopático" con o sin desprendimiento amniocorial, (IMCA 14\%) (37,38 $^{3}$ membranas prolapsadas bajo el orificio cervical externo en el embarazo de pretérmino $(\text { IMCA } 51 \%)^{39}$, desprendimiento prematuro de placenta normoinserta DPPNI, (IMCA 7\%) ) $^{15,38,40}$, pacientes sin amenaza de PP y cérvix menor de $15 \mathrm{~mm}$ medido por ultrasonografía, (IMCA 4\%) ${ }^{41,42}$,

- Datos de laboratorio: presencia de infección génito urinaria (IGU): VB o VA por $S$. agalactiae y/o infección del tracto urinario (ITU) ${ }^{7,23,24,43} \mathrm{y}$

- Hallazgos placentarios, lesiones agudas propias de infección: $\mathrm{CH}$ aguda, funisitis aguda, intervellositis $^{18-20,38,44}$

Se considera que la IBA es causa de MF en presencia de uno o más datos clínicos o de laboratorio, más lesiones inflamatorias placentarias

\section{¿Por qué el método de clasificación de MF que usa la biopsia placentaria es eficiente para identificar la causa primaria de la muerte por IBA?}

Este método es más eficiente porque permite distinguir los diferentes mecanismos que tiene la IBA para causar finalmente la muerte fetal.

\section{Evidencias}

- En una serie con 279 casos de $\mathrm{MF}^{15}$, desde las 22 semanas de gestación, se conocieron las condiciones finales de MF por IBA (45 casos) mediante el análisis de datos clínicos, de laboratorio, placentario y autopsia fetal. Las causas últimas de MF por IBA identificadas por la autopsia fueron: hipoxia fetal aguda en casos de neumonía y de sepsis con shock séptico (las condiciones más frecuentes en esta serie); e hipoxia fetal secundaria a asfixia en los casos con DPPNI y funisitis. 
detallado que abarca datos clínicos, patológicos (autopsia fetal, estudio placentario) y fisiopatológicos obtenidos a través de un riguroso proceso de estudio. Está diseñado para disminuir las muertes inexplicables usando criterios estrictos y bien definidos basados en causas originarias de $\mathrm{MF}$.

Esta herramienta, denominada "Causas Iniciales de Muerte Fetal", incluye causas conocidas de muerte y les asigna como posibles o probables según estrictos criterios de diagnóstico, basados en referencias publicadas sobre secuencias fisiopatológicas que conducen a la muerte fetal.

Se consideraron seis amplias categorías de causas de mortinatos: afecciones médicas maternas, complicaciones obstétricas, condiciones hematológicas maternas o fetales, anomalias genéticas, estructurales y cariotipicas fetales, infección placentaria, infección fetal o ambas y hallazgos patológicos placentarios.

De los métodos publicados, éste es sin duda el más eficiente, con mejores resultados y con alta calidad científica. Permite identificar la MF asociada a todos los microorganismos como agentes causales de muerte, bacterias, virus, parásitos, hongos, utilizando datos clínicos, post mortem y de patología placentaria. En el estudio para investigar la IBA como causa de MF, se incluyeron 512 mortinatos, mayores de 20 semanas, inscritos en la Red de Investigación Colaborativa de Stillbirth, en un análisis prospectivo, multicéntrico, geográfico, racial y étnicamente diverso, con población de E.U.A. Los casos tuvieron una evaluación que incluyó entrevista materna, recolección de muestras biológicas, autopsia fetal y patología placentaria, cultivos bacterianos, evaluación de sífilis y serología de parvovirus. La infección como causa de MF se identificó en 12,9\% (66/512 casos). De éstos $11,1 \%(57 / 512)$ son atribuibles a infecciones bacterianas y $1,8 \%(9 / 66)$ a infecciones no bacterianas. La MF relacionada con infección ocurrió a menor edad gestacional que aquella sin relación $(\mathrm{p}<0,001)$. El cultivo bacteriano fetal fue positivo en $53 \%$. Las bacterias predominantes fueron E. coli y S. agalactiae. Los marcadores de inflamación/ infección de la placenta fueron: corioamnionitis $76 \%$ (50/66) y funisitis $41 \%(27 / 66)$. La tinción viral fue positiva en $11 \%(7 / 66)$. El estudio de la patología placentaria fue 2,5 veces más eficiente para identificar inflamación o evidencia de infección $99 \%(\mathrm{CH} /$ funisitis, cultivo +$)$ (65/66 casos) que la autopsia fetal $39 \%$ (26/66 casos). En los casos de mortinatos relacionados con infecciones no bacterianas o por bacterias no cultivables, los microorganismos identificados fueron citomegalovirus, parvovirus, Treponema pallidum y herpes simplex

Comentario. Los datos existentes en la literatura científica sobre MF e infección es reducida y no se ha demostrado predomino de ningún microorganismo causante de muerte. En este estudio la mayoría de los casos de mortinatos relacionados con infecciones se asociaron con 
patógenos bacterianos, especialmente $S$. agalactiae y se presentaron en PPs espontáneos con edades gestacionales pre o periviables.

Recomiendan los autores reducir las infecciones para disminuir la MF y el PP espontáneo por esta causa y esperan contar prontamente con una vacuna contra $S$. agalactiae $^{45}$.

En relación a cribado de infecciones, los autores señalan: que en ausencia de evidencia de otra causa infecciosa de MF, no se recomienda la serología materna para detección sistemática de infecciones (TORCH); que el cultivo fetal positivo es insuficiente para respaldar la infección como causa de muerte en ausencia de otros hallazgos sugerentes de infección (características clínicas, patología placentaria y autopsia fetal); que existe mayor asociación de MF por infección en la población negra no hispana.

\section{- INCODE, estudio en Soweto, Sudáfrica ${ }^{14}$}

Trabajo prospectivo, observacional, en el hospital de Soweto, para investigar las causas de los mortinatos en fetos desde las 22 semanas de edad gestacional o con un peso al nacer de $500 \mathrm{~g}$ o más, mediante el método INCODE, ocupando datos clínicos, examen placentario macroscópico e histopatológico y hemocultivo fetal de muestras de cordón. Se incluyeron 298 MF. La infección placentaria o fetal fue $19,5 \%$ (58/298 casos). De éstos, $15,8 \%$ (47/298) tuvieron infección bacteriana invasora fetal y 3,7\% (11/298) infección no bacteriana. El 46,6\% (27/58) tuvo inflamación de la membrana fetal y/o de la placenta. Las bacterias más frecuentes aisladas en las infecciones invasoras causantes de la MF fueron $S$. agalactiae y E. coli. Este estudio es uno de los pocos realizados en África sobre MF por infección. De África hay muy pocos estudios sobre MF. Este es uno de las más importantes.

\section{- Método CORM en el HCSBA. Estudio uno}

Trabajo retrospectivo sobre cohorte de 29.916 nacimientos con 258 MFs desde las 22 semanas. Los datos provienen de los informes de las auditorías de mortinatos. Se aplicó el método de Clasificación Condición Obstétrica Relevante De La Muerte (CORM), que usa la biopsia placentaria, los datos clínicos y de laboratorio analizados por un obstetra y un patólogo. Se identificaron 91,5\% de las condiciones obstétricas de MF. La infección fue responsable de 30\% de los casos, IBA $26,4 \%$, ITP 3,5\% (virales, sífilis, bacterias periodontales). La causa más frecuente de esta serie fue IBA 26,4\% (68/258 casos). En esta condición, el DPPNI como causa final de muerte se presentó en uno de cada tres casos. La muerte fetal por IBA se presentó durante el embarazo y durante el parto en igual número de casos ${ }^{5}$.

El método CORM demuestra gran eficacia porque identificó 91,5\% de las condiciones originarias de MF, incluyendo la IBA, que es la condición más común de MF y reduce la frecuencia de las causas inexplicables. Es más eficiente que los métodos convencionales en detectar la IBA como causa de MF. Es reproducible, requiere estudio histopatológico de la placenta y no de autopsia fetal. Es aplicable, tanto en nacimientos de término como de pretérmino. El conocimiento de la causa de la MF, proporciona apoyo a la familia para sobrellevar el duelo y permite elaborar guías de prevención por causa.

\section{- Método CORM en el HCSBA Estudio dos ${ }^{2}$}

Trabajo retrospectivo sobre cohorte, de las MF desde las 22 semanas ocurridas en la Maternidad del HCSBA durante 10 años. Los datos provienen de los informes de las auditorías de mortinatos. Se aplicó el método de CORM, que usa la biopsia placentaria, los datos clínicos y de laboratorio analizados por un obstetra y un patólogo. Ocurrieron 56.130 nacimientos y 479 MFs. Se identificaron $93,5 \%$ de las condiciones obstétricas de MF y 6,5\% fueron inexplicables. La más frecuente fue IBA 24,9\% (119 casos). De estos 119 óbitos, 75\% y $64 \%$ fueron menores de 30 semanas y bajo 1.000 gramos, respectivamente. Esta es la frecuencia más alta reportada en la literatura médica de MF por IBA asociada con PP temprano.

En este estudio y en el anterior, a diferencia de la mayoría de los trabajos sobre mortinatos, no se consideró la autopsia fetal, para identificar a la IBA como causa de MF y podría constituir un sesgo. Sin embargo, estimamos que esto no debió ocurrir porque la autopsia no modifica la proporción de nacidos muertos inexplicables ${ }^{46-48}$ y porque el estudio placentario es más eficiente en determinar la MF por IBA ${ }^{4}$. Además, la autopsia habitualmente no se hace por la negativa frecuente de la familia, por falta de recursos y por carencia de especialistas. En cambio, el estudio placentario es aceptado por la paciente y además permite elaborar guías y normas de prevención de resultados adversos por IBA, MF PP y muerte neonatal precoz del niño prematuro ${ }^{2,4,5,15,17,21}$.

\section{Resumen y conclusiones}

Muchos niños mueren antes de nacer en todo el mundo y la IBA es causa frecuente de MF. Con los métodos convencionales de clasificación de MF que incluyen preferentemente la autopsia fetal no se ha logrado reducir la muerte por esta causa, porque reportan baja frecuencia de MF por infección y alta proporción de muertes inexplicables. Los sistemas que utilizan los datos clínicos, de laboratorio y estudio placentarios preferentemente, INCODE, CORM, son más eficientes para identificar la IBA como causa originaria de MF.

Se demostró que el estudio placentario con marcadores 
específicos de infección/inflamación $\mathrm{CH} /$ funisitis aguda es más eficiente que la autopsia fetal para identificar la IBA, que el cultivo de LA es más eficiente que el cultivo de la placenta para detectar IMCA, que la muestra de sangre de cordón es eficiente para el diagnóstico etiológico de la infección.

El conocimiento de la IBA como causa originaria de la MF ayuda a elaborar guías y normas de prevención de la MF y de otros resultados adversos asociados a esta infección ascendente, como parto prematuro ${ }^{49}$.

Los estudios presentados, constituyen un volumen de evidencia científica, que permiten recomendar el análisis de la placenta en el estudio de la causa de MF por IBA. Recomendación A (CTFPHC, SIGN) ${ }^{50}$.

Además, los resultados complementarios encontrados, permiten recomendar el análisis de los datos clínicos y de laboratorio en el estudio de MF por IBA. Recomendación $B^{50}$.

\section{Referencias bibliográficas}

1.- MacDorman M F, Gregory E C. Fetal and Perinatal Mortality: United States, 2013. Natl Vital Stat Rep. 2015; 64 (8): 1-24. PMID: 26222771.

2.- Ovalle A, Valderrama O, Alvarado A, Kakarieka E. El método de clasificación de mortinatos "Condición Obstétrica Relevante de la Muerte Fetal" (CORM) reduce la frecuencia de muerte fetal de causa inexplicable. Rev Chil Obst Ginecol. 2019; 84 (2): 91-102. http://dx.doi.org/10.4067/S071775262019000200091.

3.- The Stillbirth Collaborative Research Network Writing group. Causes of death among stillbirths. JAMA 2011; 306: 2459-68. doi: 10.1001/jama.2011.1823.

4.- Page J M, Bardsley T, Thorsten V, Allshouse A A, Varner M W, Debbink M P, et al. Stillbirth associated with infection in a diverse U.S. cohort. Obstet Gynecol. 2019 Dec; 134 (6): 1187-96. doi: 10.1097/ AOG.0000000000003515.

5.- Ovalle A, Fuentes A, Chacón V, Espinoza C, González R, Kakarieka E, et al. Método de clasificación de mortinatos según condición obstétrica relevante de la muerte fetal, en un hospital público de Chile (Método CORM) Rev Med Chile 2016; 144: 1020-28. http://dx.doi. org/10.4067/S0034-98872016000800009.

6.- Tolockiene E, Morsing E, Holst E, Herbst A, Ljungh A, Svenningsen N, et al. Intrauterine infection may be a major cause of stillbirth in Sweden. Acta Obstet Gynecol Scand. 2001; 80: 511-8. PMID: 11380286

7.- Wigglesworth J S. Monitoring perinatal mortality - A pathophysiological approach. Lancet 1980; ii: 684-6. doi: 10.1016/s01406736(80)92717-8.

8.- de Galan-Roosen A E M, Kuijpers J C, van der Straaten P J C, Merkus J M W M. Fundamental classification of perinatal death; Validation of a new classification system of perinatal death. Eur J Obstet Gynaecol Reprod Biol. 2002; 103: 30-6. doi: 10.1016/s03012115(02)00023-4.

9.- de Galan-Roosen A E, Kuijpers J C, van der Straaten P J, Merkus J M. Evaluation of 239 cases of perinatal death using a fundamental classification system. Eur J Obstet Gynecol Reprod Biol. 2002; 103 (1): 37-42. doi:10.1016/s0301-2115(02)00024-6.

10.- Gardosi J, Kady S M, McGeown P, Francis A, Tonks A. Classification of stillbirth by relevant condition at death (ReCoDe): population based cohort study. Br Med J. 2005 Nov 12; 331 (7525): 1113-7. doi. 10.1136/ bmj.38629.587639.7C.

11.- Korteweg F J, Gordijn S J, Timmer A, Erwich J J H M, Bergman K A, Bouman K, et al. The Tulip classification of perinatal mortality: introduction and multidisciplinary inter-rater agreement. Br J Obstet Gynecol. 2006; 113 (4): 393-401. doi:10.1111/j.14710528.2006.00881.x.

12.- Goldenberg R L, Muhe L, Saleem S, Dhaded S, Goudar S S, Patterson J, et al. Criteria for assigning cause of death for stillbirths and neonatal deaths in research studies in lowmiddle income countries. J Matern Fetal Neonatal Med. 2019 Jun; 32 (11): 1915-23. doi: 10.1080/14767058.2017.1419177.

13.- Dudley D J, Goldenberg R, Conway D, Silver R M, Saade G R, Varner M W, et al. Stillbirth Research Collaborative Network. A new system for determining the causes of stillbirth. Obstet Gynecol. 2010 Aug; 116 (2Pt1): 254-60. doi: 10.1097/AOG.0b013e3181e7d975.

14.- Madhi S A, Briner C, Maswime S, Mose S, Mlandu P, Chawana R, et al. Causes of stillbirths among women from South Africa: a prospective, observational study. Lancet Glob Health. 2019; 7 (4): e503-e512. doi: 10.1016/ S2214-109X(18)30541-2.

15.- Ovalle A, Kakarieka E, Correa A, Vial M T, Aspillaga C. Estudio anatomo-clínico de las causas de muerte fetal. Rev Chil Obstet Ginecol. 2005; 70: 303-12. doi: 10.4067/S0717. 75262005000500005 .

16.- Wojcieszek A M, Shepherd E, Middleton P, Gardener G, Ellwood D A, McClure E M, et al. Interventions for investigating and identifying the causes of stillbirth. Cochrane Database Syst Rev. 2018; 4 (4): CD012504. Published 2018 Apr30. doi: 10.1002/14651858.CD012504. pub2.

17.- Ovalle A, Martínez M A, Giglio M S,
Poblete J P, Fuentes A, Gómez R, et al. Microbiología aislada en la rotura prematura de membranas de pretérmino. Relación con morbilidad infecciosa materna neonatal e intervalo rotura de membranas-parto. Rev Chil Obstet Ginecol. 1995; 60: 252-62.

18.- Romero R, Gómez-López N, Winters A D, Jung E, Chamán M, Bieda J, et al. Evidence that intra-amniotic infections are often the result of an ascending invasion-a molecular microbiological study. J Perinat Med. 2019; 47 (9): 915-31. doi:10.1515/jpm-20190297.

19.- Ovalle A, Martínez M A, Kakarieka M E, Gómez R, Torres J, Fuentes A, et al. Histopatología de la placenta en la rotura prematura de membranas de pretérmino. Relación con la microbiología aislada y con los resultados materno-neonatales. Rev Med Chile 1998; 126: 930-42. PMID: 9830745.

20.- Ovalle A, Gómez R, Martínez M A, Kakarieka E, Fuentes A, Aspillaga C, et al. Invasión microbiana de la cavidad amniótica en la rotura de membranas de pretérmino. Resultados materno-neonatales y patología placentaria según microorganismo aislado. Rev Med Chile 2005; 133: 51-61. http://dx.doi. org/10.4067/ S0034-98872005000100007.

21.- Han X, Du H, Cao Y, Zhang Y, Zhang J, Zhang L, et al. Association of histological and clinical chorioamnionitis with perinatal and neonatal outcome. J Matern Fetal Neonatal Med. 2019 May 31: 1-9. doi: 10.1080/14767058.2019.1618824.

22.- Hillier S L, Krohn M A, Kiviat N B, Watts H, Eschenbach A. Microbial causes and neonatal outcomes associated with chorioamnion infection. Am J Obstet Gynecol. 1991; 165: 955-61. doi: 10.1016/00029378(91)90447-Y.

23.- Nan C, Dangor Z, Cutland C L, Edwards M S, Madhi S A, Cunnington MC. Maternal group B streptococcus-related stillbirth: a systematic review. Br J Obstet Gynecol 2015; 122: 1437 45. doi: 10.1111/1471-0528.13527.

24.- Seale A C, Blencowe H, Bianchi-Jassir F, Embleton N, Bassat Q, Ordi J, et al. Stillbirth with group B Streptococcus disease worldwide: systematic review and meta-analysis. Clin 
Infect Dis. 2017; 65: S125-S132. doi: 10.1093/ cid/cix 585 .

25.- Ovalle A, Gamonal J, Martínez M A, Silva N, Kakarieka E, Fuentes A, et al. Relación entre enfermedad periodontal, infección bacteriana ascendente y patología placentaria con parto prematuro. Rev Med Chile 2009; 137: 513-23. http://dx.doi.org/10.4067/S003498872009000400008 .

26.- Gauthier S, Tétu A, Himaya E, Morand M, Chandad F, Rallu F, et al. The origin of Fusobacterium nucleatum involved in intraamniotic infection and preterm birth. J Matern Fetal Neonatal Med. 2011 Nov; 24 (11): 1329 32. doi: 10.3109/14767058.2010.550977.

27.- Murphy T F. Haemophilus species, including Haemophilus influenzae and H. ducreyi (Chancroid). En Mandell, Douglas \& Bennett"s Principles and Practice of Infectious Diseases. Bennett Md Macp, John E.; Dolin Md, Raphael; Blaser Md, Martin J., eds. Elsevier, New York; 2019, 9th. Ed Chapter 225. pp: 2743-52.e.

28.- Martínez M A, Ovalle A, Ulloa M T y Vidal R. Role of Haemophilus influenzae in intraamniotic infection in patients with preterm rupture of membranes. Eur J Clin Microb \& Infect Dis. 1999; 18: 890-93. doi: 10.1007/ s100960050425.

29.- Ovalle A, García M, Oda F, Alvarado S, Martínez M A. Meningitis neonatal precoz causada por transmisión vertical de Escherichia coli productora de beta-lactamasa de espectro extendido en parto prematuro con rotura prematura de membranas. Rev Chil Obstet Ginecol 2017; 82 (6): 621-5. doi: 10.4067/ S0717-75262017000600621.

30.- Ovalle A, Martínez M A, Kakarieka E, García Mirna, Salinas A. Sepsis neonatal precoz causada por transmisión vertical de Morganella morganii, en un embarazo de término. Rev Med Chile 2009; 137: 1201-4. doi:10.4067/S003498872009000900010

31.- Gómez R, Romero R, Ghezzi F, Yoon B H, Mazor M, Berry S M. The fetal inflammatory response syndrome. Am J Obstet Gynecol. 1998; 179: 194-202 PMID: 9704787.

32.- Bracci R, Buonocore G. Chorioamnionitis: a risk factor for fetal and neonatal morbidity. Biol Neonate 2003; 83 (2): 85-96. doi: 10.1159/000067956.

33.- Schendel D E, Schuchat A, Thorsen P. Public health issues related to infection in pregnancy and cerebral palsy. Ment Retard Dev Disabil Res Rev. 2002; 8: 39-45. doi: 10.1002/ mrdd.10011.

34.- Chaiworapongsa T, Romero R, Kim J C, Kim Y M, Blackwell S C, Yoon B H, et al. Evidence for fetal involvement in the pathologic process of clinical chorioamnionitis. Am J Obstet Gynecol. 2002; 186: 1178-82. doi: 10.1067/ mob.2002.124042.

35.- Pacora P, Chaiworapongsa T, Maymon E, Kim Y M, Gomez R, Yoon B H, et al. Funisitis and chorionic vasculitis: the histological counterpart of the fetal inflammatory response syndrome. J Matern Fetal Neonatal Med. 2002; 11: 18-25. doi: 10.1080/jmf.11.1.18.25.

36.- Romero R, Gómez R, Ghezzi F, Yoon B H, Mazor M, Edwin S S, et al. A fetal systemic inflammatory response is followed by the spontaneous onset of preterm parturition. Am J Obstet Gynecol. 1998; 179: 186-93. doi: 10.1016/s0002-9378(98)70271-6.

37.- Gómez R, Romero R, Nien J K, Medina L, Carstens M, Kim Y M, et al. Idiopathic vaginal bleeding during pregnancy as the only clinical manifestation of intrauterine infection. J Matern Fetal Neonatal Med. 2005; 18 (1): 31-7. doi: 10.1080/14767050500217863.

38.- Ovalle A, Kakarieka E, Rencoret G, Fuentes A, del Río M J, Morong C, et al. Factores asociados con el parto prematuro entre 22 y 34 semanas en un hospital público de Santiago. Rev Med Chile 2012; 140: 19-29. doi. 10.4067/ S0034- 98872012000100003.

39.- Romero R, González R, Sepúlveda W, Brandt F, Ramírez M, Sorokin Y, et al. Infection and labor. VIII. Microbial invasion of the amniotic cavity in patients with suspected cervical incompetence: prevalence and clinical significance. Am J Obstet Gynecol. 1992; 167 1086-91. PMID: 1415396.

40.- Ovalle A, Kakarieka E, Díaz M, García Huidobro T, Acuña M J, Morong K, et al. Mortalidad perinatal en el parto prematuro entre 22 y 34 semanas en un hospital público de Santiago, Chile. Rev Chil Obstet Ginecol. 2012; 77: 263-70. doi: 10.4067/S0717. 75262012000400005.

41.- Vaisbuch E, Hassan S S, Mazaki-Tovi S, Nhan-Chang C L, Kusanovic J P, Romero R, et al. Patients with an asymptomatic short cervix $(<15 \mathrm{~mm})$ have a high rate of subclinical intraamniotic inflammation: implications for patient counseling. Am J Obstet Gynecol. 2010; 202: 433.e1-8. doi: 10.1016/j.ajog.2010.02.007.

42.- Hassan S, Romero R, Hendler I, Gómez R, Khalek N, Espinoza J, et al. A sonographic short cervix as the only clinical manifestation of intra-amniotic infection. J Perinat Med. 2006; 34: 13-19. doi:10.1515/JPM.2006.002.

43.- Álvarez J R, Fechner A J, Williams S F, Ganesh V L, Apuzzio J J. Asymptomatic bacteriuria in pregestational diabetic pregnancies and the role of group B Streptococcus. Am J Perinatol. 2010; 27: 231-4. doi: 10.1055/s-0029-1239485.

44.- Rezeberga D, Lazdane G, Kroica J, Sokoloval, Donders G G. Placental histological inflammation and reproductive tract infections in a low risk pregnant population in Latvia. Acta Obstet Gynecolog. Scand 2008; 87: 36065. doi: 10.1080/00016340801936487.

45.- Group B Streptococcus Vaccine Development Technology Roadmap. Priority activities for development, testing, licensure and global availability of group B Streptococcus vaccines. Geneva, Switzerland: World Health Organization; 2017. https://apps.who.int/iris/ handle/10665/258704.

46.- Pasztor N, Kereszturi A, Kozinszky Z, Pál A. Identification of causes of stillbirth through autopsy and placental examination reports. Fetal Pediatr Pathol. 2014; 33 (1): 49-54. doi: 10.3109/15513815.2013.850132.

47.- Man J, Hutchinson J C, Heazell A E, Ashworth M, Levine S, Sebire N J. Stillbirth and intrauterine fetal death: factors affecting determination of cause of death at autopsy. Ultrasound Obstet Gynecol. 2016; 48 (5): 56673. doi: 10.1002/uog. 16016.

48.- Kaschula R O. The pediatric autopsy in Africa. Arch Pathol Lab Med. 2013; 137 (6): 756-66. doi: 10.5858/arpa.2011-0589-RA.

49.- Ovalle A, Martínez M A, Figueroa J. ¿Se puede prevenir el parto prematuro por infección bacteriana ascendente y sus resultados adversos en los hospitales públicos de Chile? Artículo de revisión. Rev Chilena Infectol. 2019; 36 (3): 358-68, doi: 10.4067/S071610182019000300358.

50.- Manterola C, Asenjo-Lobos C, Otzen T. Hierarchy of evidence: levels of evidence and grades of recommendation from current use. Rev Chilena Infectol. 2014; 31:705-18. doi: 10.4067/S0716-10182014000600011. 